\title{
Fresh Garlic Extract Enhances the Antimicrobial Activities of Antibiotics on Resistant Strains in Vitro
}

\author{
Guoliang Li ${ }^{1}$; Xudong Ma ${ }^{1}$; Lisha Deng ${ }^{2}$; Xixi Zhao ${ }^{2}$; Yuejiao Wei ${ }^{1}$; Zhongyang Gao ${ }^{2}$; Jing Jia ${ }^{1}$; \\ Jiru Xu ${ }^{2, ;} ;$ Chaofeng Sun ${ }^{1}$ \\ ${ }^{1}$ The First Affiliated Hospital, Xi'an Jiaotong University Health Science Center, Xi'an, China \\ ${ }^{2}$ Department of Pathogen Biology and Immunology, College of Basic Medicine, Xi'an Jiaotong University Health Science Center, Xi'an, China \\ ${ }^{*}$ Corresponding author: Jiru Xu, Department of Pathogen Biology and Immunology, College of Basic Medicine, Xi'an Jiaotong University Health Science Center, Xi'an, China. \\ Tel/Fax: +86-2985323805, E-mail: xujiru@mail.xjtu.edu.cn
}

Received: September 13, 2013; Revised: April 13, 2014; Accepted: April 20, 2014

\begin{abstract}
Background: Infections caused by strains with multi-drug resistance are difficult to treat with standard antibiotics. Garlic is a powerful remedy to protect against infections of many bacteria, fungi and viruses. However, little is known about the potentials of fresh garlic extract (FGE) to improve the sensitivity of multi-drug resistant strains to antibiotics.

Objectives: In this study, we used the disk diffusion method to investigate the antimicrobial activities of FGE and the combination of antibiotics with FGE, on methicillin-resistant Staphylococcus aureus (MRSA), Pseudomonas aeruginosa and Candida albicans, to evaluate the interactions between antibiotics and FGE.

Materials and Methods: Clinical isolates were isolated from clinical specimens obtained from the inpatients at the First Affiliated Hospital of Xi'an Jiaotong University Health Science Center. The isolates consisted of MRSA, $(\mathrm{n}=30)$, C. albicans $(\mathrm{n}=30)$ and $P$. aeruginosa $(\mathrm{n}$ =30). Quality control for CLSI (Clinical and Laboratory Standards Institute) disk diffusion was performed using S. aureus ATCC®25923, C. albicans ATCC®90028 and P. aeruginosa ATCC®27853. The 93 microorganisms were divided into four groups in a factorial design: control (deionized water), FGE, antibiotics without FGE, and antibiotics with FGE. Next, antibacterial activity was evaluated by measuring the diameter of inhibition zones according to performance standards for antimicrobial susceptibility testing of the Clinical and Laboratory Standards Institute (CLSI, formerly NCCLS).

Results: Fresh garlic extract displayed evident inhibition properties against C. albicans and MRSA, yet weak inhibition properties against P. aeruginosa. Additionally, FGE showed the potential to improve the effect of antibiotics on antibiotic resistant pathogens. The synergism of fluconazole and itraconazole with FGE on C. albicans yielded larger sized inhibition zones compared with fluconazole and itraconazole without FGE $(\mathrm{P}<0.01)$. The factorial analysis represents intense positive interaction effects $(\mathrm{P}<0.01)$. The synergism of cefotaxime and ceftriaxone with FGE on $P$. aeruginosa yielded larger sized inhibition zones than cefotaxime and ceftriaxone without FGE $(\mathrm{P}<0.01)$. The factorial analysis represents intense positive interaction effects $(\mathrm{P}<0.01)$.

Conclusions: The results suggest that FGE can improve the antibiotic sensitivity of these pathogens to some antibiotics.
\end{abstract}

Keywords: Garlic; Drug Resistance, Microbial; Candida albicans; Methicillin-Resistant Staphylococcus aureus; Pseudomonas aeruginosa

\section{Background}

With antibacterial drugs being widely used in clinical settings, many microorganisms, especially methicillinresistant Staphylococcus aureus (MRSA), Pseudomonas aeruginosa and Candida albicans, have adapted to synthetic antibiotics and become highly resistant to these drugs over time (1-4). Microorganisms with multi-drug resistance now cause thousands of deaths throughout the world each year $(1,3)$. Although some of these organisms can live harmlessly in humans and are carried in the nasal passage and on the skin, they can cause fatal infection in hospitals and nursing homes, where patients with open wounds, invasive devices and immunodeficiency are at higher risk of infection than healthy people $(5,6)$.
Furthermore, resistance does make the infection more difficult to treat with standard antibiotics and thus more dangerous $(7,8)$. Therefore, the continuing spread of multi-drug resistant strains and the increased abuse of antibiotics highlight the need for alternative agents.

Garlic has been found to help prevent many diseases. Numerous modern studies confirm that garlic has definite antibiotic properties and is effective against a wide spectrum of bacteria, fungi and viruses $(9,10)$. In addition, the antimicrobial activities of garlic are linked to the presence of some bioactive compounds (11). Moreover, many studies have demonstrated that garlic can be more effective as a broad-spectrum antibiotic compared

Copyright (C) 2015, Ahvaz Jundishapur University of Medical Sciences. This is an open-access article distributed under the terms of the Creative Commons Attribution-NonCommercial 4.0 International License (http://creativecommons.org/licenses/by-nc/4.0/) which permits copy and redistribute the material just in noncommercial usages, provided the original work is properly cited. 
with conventional antibiotics. However, most previous studies have only focused on the antimicrobial activities of garlic and garlic-derived organ sulfur compounds or the difference between garlic or garlic-derived organ sulfur compounds and standard antibiotics, while little is known about the potential of fresh garlic extract (FGE) to improve the susceptibility of multi-drug resistant strains to conventional antibiotics.

\section{Objectives}

The present study aimed to investigate the antimicrobial activities of FGE and the combination of FGE and conventional antibiotics on common clinical strains, including MRSA, multi-drug resistant $P$. aeruginos $a$ and $C$. albicans, to evaluate the interactions between antibiotics and FGE.

\section{Materials and Methods}

\subsection{Fresh Garlic Extract}

Fresh garlic bulbs (Chinese white garlic) were purchased from the Northwest Agriculture Forestry University (Yangling, China). Peeled garlic bulbs (100 g) were blended in $50 \mathrm{~mL}$ sterile distilled water. The mixture was crushed finely using a juicer. The resulting paste was centrifuged at $3000 \mathrm{rmp}$ for 30 minutes and the supernatant was then sterilized by a filter $(0.2 \mu \mathrm{m}$ pore size, Steriflip, Millipore). The final concentration of FGE in aqueous solution was determined to be $40.7 \%(\mathrm{w} / \mathrm{v})$ by subtracting the weight of the precipitate from the weight of the original peeled garlic bulbs. The FGE was stored in $1.5 \mathrm{~mL} \mathrm{mi-}$ cro test tubes at $-20^{\circ} \mathrm{C}$ until used.

\subsection{Microbial Strains}

A total of 90 clinical isolates and three control strains were used. The clinical isolates were isolated from clinical specimens obtained from the inpatients at the First Affiliated Hospital of Xi'an Jiaotong University Health Science Center. The isolates consisted of MRSA, $(n=30), C$. albicans $(\mathrm{n}=30)$ and $P$. aeruginosa $(\mathrm{n}=30)$. Quality control for CLSI (Clinical and Laboratory Standards Institute) disk diffusion was performed using S. aureus ATCC®25923, C. albicans ATCC® 90028 and P. aeruginosa ATCC® 27853. All isolates were identified at the strain level using the Vitek 2 automatic system (Bio Merieux Company, Marcy l'Etoile, France) and showed multiple antibiotic resistances, and had no apparent epidemiological connection. The isolated strains were then transported to nutrient agar slants and stored at $2-8{ }^{\circ} \mathrm{C}$ until use. Purity of the organisms was checked at regular intervals by plating and staining. The 93 microorganisms were divided into four groups in a factorial design: control (deionized water), FGE, antibiotics without FGE, and antibiotics with FGE.

\subsection{Antimicrobial Susceptibility Test}

The following antibiotic-containing paper disks (Oxoid, UK) were used: $8 \mu \mathrm{g}$ itraconazole (I), $15 \mu \mathrm{g}$ fluconazole (F), $30 \mu \mathrm{g}$ cefoxitin (FOX), $1 \mu \mathrm{g}$ oxacillin (OX), $100 \mu \mathrm{g}$ piperacillin (PRL), $30 \mu \mathrm{g}$ cefotaxime (CTX), $5 \mu \mathrm{g}$ levofloxacin (LEV), $30 \mu \mathrm{g}$ ceftriaxone (CRO), $10 \mu \mathrm{g}$ ampicillin (AMP) and $30 \mu \mathrm{g}$ cefazolin (KZ). The 0.5 McFarland standard suspension of germ solution was inoculated onto Mueller Hinton agar (Becton Dickinson, Sparks, MD, USA) supplemented with $2 \%$ glucose and $0.5 \mathrm{~g} / \mathrm{ml}$ methylene blue. Fifteen minutes after the agar absorbed the bacterial suspension; the antibiotic-containing paper disk on Muller-Hinton agar plates was placed onto the surface of the inoculated plate (90 mm) with sterile forceps to investigate the antibiotic activity. Next, $50 \mu \mathrm{L}$ of FGE was pipetted on the Whatman No. 1 filter paper discs (6 $\mathrm{mm}$ in diameter) and antibioticcontaining paper disk to evaluate the activities of FGE and the combinative activities of antibiotics and FGE. Meanwhile, $50 \mu \mathrm{L}$ of sterile distilled water was also pipetted onto Whatman No. 1 filter paper discs as the control. The plates were left on a flat bench for one hour after the paper disk absorbed the solution. Inoculated plates were then incubated at $37^{\circ} \mathrm{C}$ for 24 hours. The antibacterial activity was then evaluated by measuring the diameter of inhibition zones according to Performance Standards for Antimicrobial Susceptibility Testing of The Clinical and Laboratory Standards Institute (CLSI, formerly NCCLS).

\subsection{Reproducibility and Statistics}

All measurements were repeated three times and each strain was examined at least three separate times. Statistical analysis was conducted using the SPSS software version 18.0 (SPSS Inc, Chicago IL). Means that were significantly different were separated using the one-way ANOVA. The factorial experiment was analyzed to evaluate the main effects and interactions between FGE and antibiotics; data were analyzed by two-way ANOVAs using the same program. P values of 0.05 were considered to be significant.

\section{Results}

\subsection{Candida albicans}

The antifungal susceptibility of C. albicans to F and I with FGE and without FGE is summarized in Figure 1. The ranking was as follows, I with FGE $>$ FGE $>$ I without FGE $>$ control; and F with FGE $>$ FGE $>$ F without FGE $>$ control. The data showed that I and F with FGE could lead to an increase in the size of the inhibition zones against $C$. albicans compared with I and $\mathrm{F}$ without FGE $(\mathrm{P}<0.01)$. The factorial analysis represents intense positive interaction effects $(P<0.01)$ (data is not shown). The fungistatic activity of I and F was dramatically enhanced by addition of FGE, and the synergism of I and F with FGE yielded an obvious increase in the size of inhibition zones, $29.0 \mathrm{~mm}$ and $30.5 \mathrm{~mm}$, respectively (Figure 1). 
Figure 1. In Vitro Antifungal Activity of Fluconazole and Itraconazole With and Without Fresh Garlic Extract Against Candida albicans

\section{A}
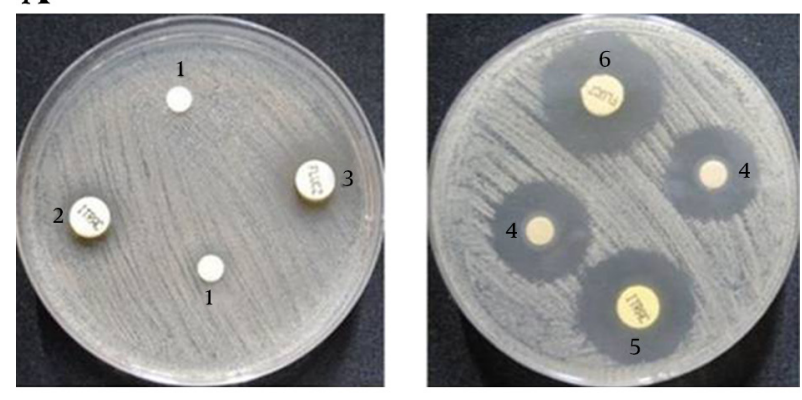

B

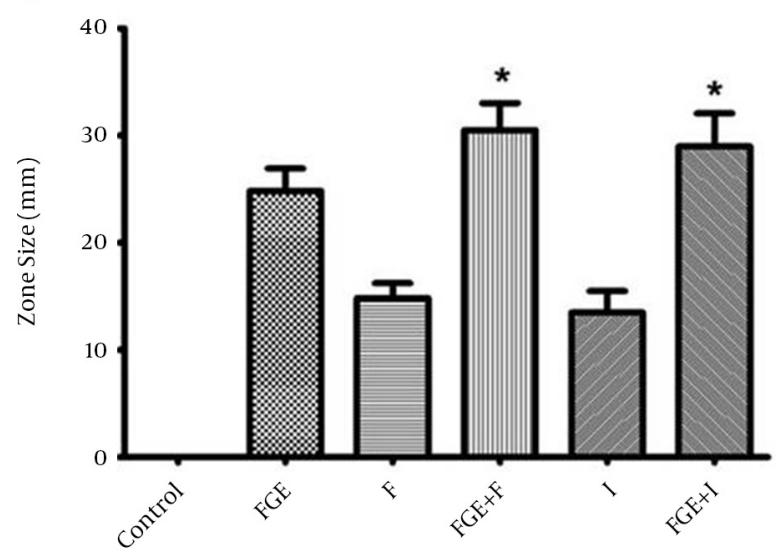

(A) Representative zones of antibacterial activity of $\mathrm{F}$ and I with and without FGE against $C$. albicans in vitro. 1, control; 2 , I; 3, F; 4, FGE; 5, I + FGE; 6, F + FGE. (B) The analysis of antibacterial activity of $\mathrm{F}$ and I with and without FGE against $C$. albicans in vitro. ${ }^{*} P$ value of $<0.05$ indicates a significant difference from the respective antibiotic without FGE.

\subsection{Methicillin-Resistant Staphylococcus aureus}

The antibacterial susceptibility of MRSA to FOX, OX and PRL with and without FGE is summarized in Figure 2. The ranking was as follows: FGE with FOX $>$ FGE $>$ FOX without FGE $>$ control; and FGE with OX $>$ FGE $>$ OX without FGE $>$ control; FGE with PRL $>$ FGE $>$ PRL without FGE $>$ control. Although FOX, OX and PRL with FGE produced larger sized inhibition zones against MRSA compared with FOX, OX and PRL without FGE $(\mathrm{P}<0.01)$, the factorial analysis indicated no intense positive interaction effects $(\mathrm{P}>0.05)$ (data is not shown).

\subsection{Pseudomonas aeruginosa}

The antibacterial susceptibility of $P$. aeruginosa to CTX, LEV, CRO, KZ and AMP with and without FGE is summarized in Figure 3. The ranking was as follows: FGE with CTX $>$ FGE $>$ CTX without FGE $>$ control; FGE with LEV
Figure 2. In Vitro Antifungal Activity of Cefoxitin, Oxacillin and Piperacillin With and Without Fresh Garlic Extract Against Methicillin Resistant Staphylococcus aureus

A

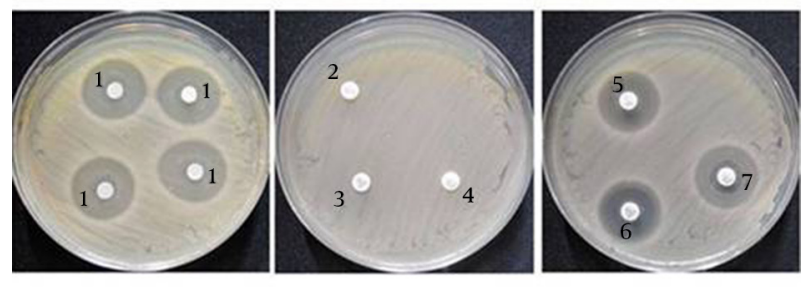

B

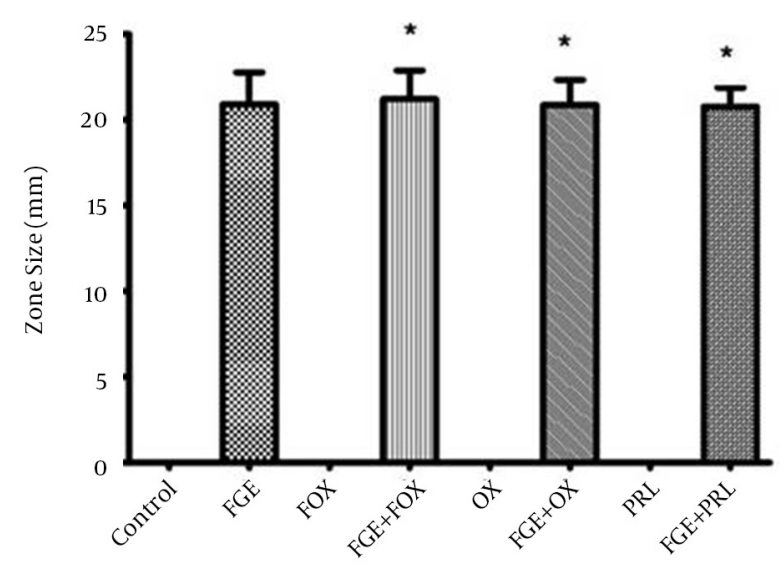

(A) Representative zones of antibacterial activity of FOX, OX and PRL with and without FGE against MRSA in vitro;1, FGE; 2, OX; 3, FOX; 4, PRL; 5, OX + FGE; 6, FOX + FGE; 7, PRL+FGE. (B) The analysis of antibacterial activity of FOX, OX and PRL with and without FGE against MRSA in vitro. ${ }^{*} \mathrm{P}$ values of $<0.05$ indicate significant difference from the respective antibiotic without FGE.

$>$ FGE $>$ LEV without FGE $>$ control; FGE with $\mathrm{CRO}>$ FGE $>$ CRO without FGE $>$ control; FGE with $\mathrm{KZ}>$ FGE $>$ $\mathrm{KZ}$ without FGE $>$ control; FGE with AMP $>$ FGE $>$ AMP without FGE $>$ control. The data showed that CTX, LEV, CRO, KZ and AMP with FGE could produce larger sized inhibition zones against $P$. aeruginosa compared with CTX, LEV, CRO, KZ and AMP without FGE. The anti-microbial activity of CTX and CRO was dramatically enhanced by addition of FGE, CTX and CRO with FGE and yielded larger sized inhibition zones compared with CTX and CRO without FGE (Figure 3). The factorial analysis represents an intense positive interaction effect between FGE and CTX and between FGE and CRO (P<0.0 1) (data is not shown). Furthermore, LEV, KZ and AMP with FGE could also produce larger sized inhibition zones against $P$. aeruginosa compared with LEV, KZ and AMP without FGE. However, the factorial analysis indicated no intense positive interaction effects $(\mathrm{P}>0.05)$ (data is not shown). 
Figure 3. In Vitro Antifungal Activity of Cefoxitin, Levofloxacin, Ceftriaxone, Cefazolin and Ampicillin With and Without Fresh Garlic Extract Against Pseudomonas aeruginosa
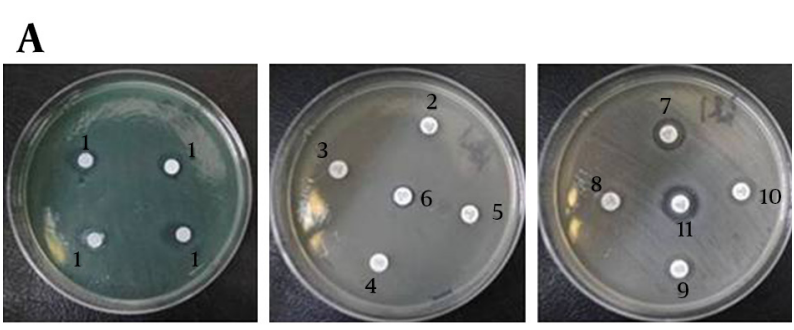

\section{B}

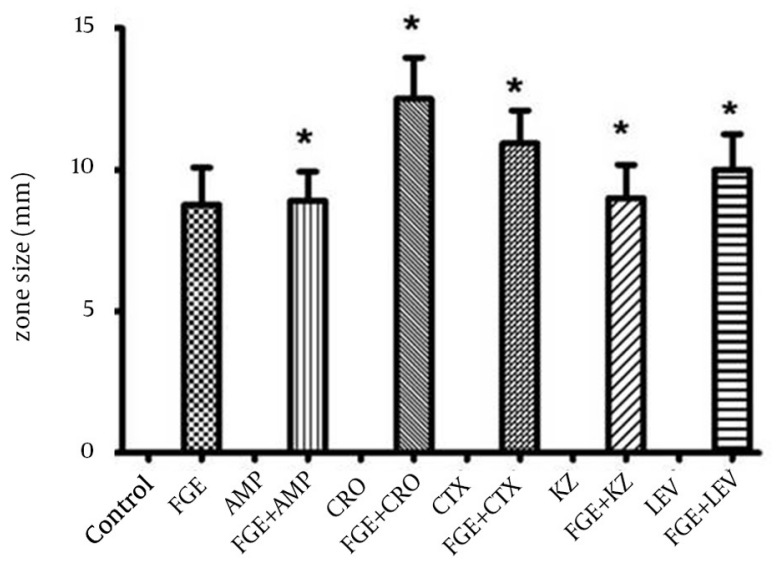

(A) Representative zones of antibacterial activity of FGE against $P$. aeruginosa in vitro; 1 , FGE; 2, CRO; 3, AMP; 4, KZ; 5, LEV; 6, CTX; 7, CTX; 8, AMP; 9, KZ; $10, \mathrm{LEV} ; 11, \mathrm{CRO}$. (B) The analysis of antibacterial activity in vitro of FOX, LEV $\mathrm{CRO}, \mathrm{KZ}$ and AMP with and without FGE against $P$. aeruginosa. ${ }^{*} \mathrm{P}$ values of $<0.05$ indicate significant difference from the respective antibiotic without FGE.

\section{Discussion}

In this study, we found that FGE displayed inhibition properties against C. albicans and MRSA and weak inhibition properties against $P$. aeruginosa. In addition, FGE could help improve the antibiotic susceptibility of these strains to some traditional antibiotics. Fresh Garlic Extract may be a candidate for the treatment of infections by multi-drug resistant strains. Our results suggest that FGE helps improve the antibiotic resistance of pathogens to some antibiotics. With the increased abuse of synthetic antibiotics and the continuing spread of strains with multi-drug resistance, the need for alternative agents is urgent. Garlic has been demonstrated to be a powerful remedy to protect against infections of many bacteria, fungi and viruses (12-14). Of all its reputed benefits, one significant advantage of garlic is its effectiveness against nosocomial strains that frequently display above average resistance to many antibiotics. Garlic contains various active components that work in complex ways. Some of these components can work together in the body to pro- tect against infections. Of all the biotical ingredients, allicin, an organ sulfur compound, is regarded as the paramount antibacterial agent in crushed garlic extracts and exhibits protective effects against attacks by pests $(14,15)$. However, allicin is rapidly oxidized, unstable and volatile, meaning it rapidly breaks down after raw garlic is cracked. It has been reported that garlic extract has more potent anti-staphylococcal activity than an equal amount of allicin. This may be because a water-based extract of garlic stabilizes allicin, at least partially, due to the hydrogen bonding between water and the reactive oxygen atom in illicit that lessens its instability and/or there may be water-soluble ingredients in cracked garlic that destabilize the molecule.

Systemic fungal infections induced by C. albicans have emerged as the main criminal of morbidity and mortality in immunocompromised patients (1). Some researchers reported on the antifungal activity of garlic in vitro against $C$. albicans $(9,10)$. In this study, C. albicans was resistant to $\mathrm{F}$ and I without FGE but susceptible to FGE. Fresh garlic extracts showed a powerful inhibitory effect against $C$. albicans compared with $\mathrm{F}$ and I; the fungistatic activity of F and I was dramatically enhanced by addition of FGE. The factorial analysis of F or I and FGE indicated intense positive interaction effects $(\mathrm{P}<0.01)$. Thus it can be suggested that FGE can distinctly improve the sensitivity of C. albicans to F or I. An et al. (16) suggested that allicin could enhance the activity of AmB against $C$. albicans in vitro and in vivo. Another study showed that a combination of $\mathrm{F}$ and allicin exhibited a good synergism against C. albicans. Some underlying mechanisms have been suggested by previous studies. Low et al. (10) found that garlic and its bioactive ingredients could suppress hyphae generation and affect the expression level of SIR2 gene. Yousuf et al. (17) affirmed that both diallyl sulfide (DAS) and diallyl disulfide (DADS) in garlic significantly inhibited proteinase, phospholipase secretion and dimorphism in C. albicans.

Methicillin-resistant Staphylococcus aureus is often considered to be as a "superbug" (12). It was estimated that the number of MRSA infections in hospitals has increased significantly and the annual deaths from MRSA infections are even more than $\operatorname{AIDS}(18,19)$. Garlic has been scientifically proven to be a powerful natural antibiotic against MRSA infections $(12,14)$. Ingredients in fresh garlic, other than illicit, have strong natural antibiotic effects (12). Garlic extract, DAS and DADS provide powerful protective activity against MRSA by affecting the pathogen distribution and plasma levels of pro-inflammatory cytokines, endothelial injury-associated proteins, and coagulation and anti-coagulation factors as well as lipid oxidation levels, and by boosting the immune system. In this study, FGE produced a strong antibacterial effect on all MRSA resistant to standard antibiotics, FOX, OX and PRL. However, the factorial analysis of FOX, OX or PRL and FGE indicated no positive interaction effects $(P>0.05)$; there exists no FGE antibiotic resistance-modifying activ- 
ity against MRSA. The antibacterial effect of the combination of FOX, OX or PRL and FGE is only attributed to FGE.

Pseudomonas aeruginosa contributes to chronic opportunistic infections, which can be fatal for immunocompromised patients and the elderly $(5,20,21)$. Biofilms of $P$. aeruginosa protect these strains from adverse environmental factors and enable the unique ability of $P$. aeruginosa to evade host innate immune defenses and the intrinsic resistance to many antibiotics $(20,22,23)$. The antimicrobial activity of garlic against $P$. aeruginosa has been widely recognized. Garlic-treated biofilms were susceptible to both tobramycin and polymorphonuclear leukocytes (PMNs) grazing. Furthermore, the PMNs incubated with garlic-treated biofilms showed an increase in respiratory burst activation. The garlic treatment initially provoked a higher degree of inflammation and significantly improved clearing of the infecting bacteria (24).

In our experiment, the interaction effect of CTX, LEV, CRO, KZ and AMP with FGE was evaluated (Figure 3). The data showed that CTX, LEV, CRO, KZ and AMP with FGE could produce larger sized inhibition zones against $P$. aeruginosa compared with CTX, LEV, CRO, KZ and AMP without FGE. The factorial analysis indicated an intense positive interaction effect between FGE and CTX or CRO $(\mathrm{P}<0.01$ ) (data is not shown). Although the LEV, KZ and AMP with FGE could produce larger sized inhibition zones against $P$. aeruginosa compared with LEV, KZ and AMP without FGE, the factorial analysis indicated no intense positive interaction effects $(\mathrm{P}>0.05)$ (dates is not shown). The combination failed to efficiently inhibit the bacteria and the factorial analysis indicated no intense positive interaction effects $(P>0.05)$. These findings are not in accordance with previous studies (24). This may be due to the variability of the strains or differences among species of garlic.

In this study, the results indicated that FGE has inhibition properties against C. albicans and MRSA but weak inhibition properties against $P$. aeruginosa, while it had the potential to improve the effect of antibiotics on antibiotic resistant pathogens. Fresh Garlic Extract may be used to aid the treatment of infections from multi-drug resistant strains. In addition, further efforts are needed to elucidate the molecular mechanisms underlying the synergistic effect between antibiotics and FGE in vitro.

\section{Acknowledgements}

The authors acknowledge Ning Zhang, the First Affiliated Hospital, Xi'an Jiaotong University Health Science Center, Long Mei and the Department of Pathogen Biology and Immunology. The Xi'an Jiaotong University Health Science Center is further acknowledged for the generous supply of tested strains.

\section{Authors' Contributions}

Study concept and design: Guoliang Li, Xudong Ma, Lisha Deng, Xixi Zhao, Yuejiao Wei, Zhongyang Gao, Jing Jia,
Chaofeng Sun and Jiru Xu. Analysis and interpretation of data: Guoliang Li, Xudong Ma, Lisha Deng, Xixi Zhao, Yuejiao Wei, Zhongyang Gao, Jing Jia, Chaofeng Sun and Jiru Xu. Drafting of the manuscript: Xudong Ma, Chaofeng Sun, and Jiru Xu. Critical revision of the manuscript for important intellectual content: Chaofeng Sun, and Jiru Xu. Statistical analysis: Xudong Ma.

\section{Funding/Support}

This research was supported by a faculty grant from the National College Student Innovative Experiment Project of China (610734).

\section{References}

1. Mane A, Gaikwad S, Bembalkar S, Risbud A. Increased expression of virulence attributes in oral Candida albicans isolates from human immunodeficiency virus-positive individuals. J Med Microbiol. 2012;61(Pt 2):285-90.

2. Balasubramanian D, Kong KF, Jayawardena SR, Leal SM, Sautter RT, Mathee K. Co-regulation of \{beta\}-lactam resistance, alginate production and quorum sensing in Pseudomonas aeruginosa. $J$ Med Microbiol. 2011;60(Pt 2):147-56.

3. Datta P, Gulati N, Singla N, Rani Vasdeva H, Bala K, Chander J, et al. Evaluation of various methods for the detection of meticillinresistant Staphylococcus aureus strains and susceptibility patterns. J Med Microbiol. 2011;60(Pt 11):1613-6.

4. Watamoto T, Samaranayake LP, Egusa H, Yatani H, Seneviratne CJ. Transcriptional regulation of drug-resistance genes in Candida albicans biofilms in response to antifungals. J Med Microbiol. 2011;60(Pt 9):1241-7.

5. Strateva T, Yordanov D. Pseudomonas aeruginosa - a phenomenon of bacterial resistance. J Med Microbiol. 2009;58(Pt 9):1133-48.

6. Patel M, Shackleton JT, Coogan MM. Effect of antifungal treatment on the prevalence of yeasts in HIV-infected subjects. J Med Microbiol. 2006;55(Pt 9):1279-84.

7. Smith K, Hunter IS. Efficacy of common hospital biocides with biofilms of multi-drug resistant clinical isolates.J Med Microbiol. 2008;57(Pt 8):966-73.

8. De Groote VN, Fauvart M, Kint CI, Verstraeten N, Jans A, Cornelis $\mathrm{P}$, et al. Pseudomonas aeruginosa fosfomycin resistance mechanisms affect non-inherited fluoroquinolone tolerance. J Med Microbiol. 2011;60(Pt 3):329-36.

9. Shuford JA, Steckelberg JM, Patel R. Effects of fresh garlic extract on Candida albicans biofilms. Antimicrob Agents Chemother. 2005;49(1):473.

10. Low CF, Chong PP, Yong PV, Lim CS, Ahmad Z, Othman F. Inhibition of hyphae formation and SIR2 expression in Candida albicans treated with fresh Allium sativum (garlic) extract. $J$ Appl Microbiol. 2008;105(6):2169-77.

11. Tsao SM, Yin MC. In-vitro antimicrobial activity of four diallyl sulphides occurring naturally in garlic and Chinese leek oils. J Med Microbiol. 2001;50(7):646-9.

12. Tsao SM, Liu WH, Yin MC. Two diallyl sulphides derived from garlic inhibit meticillin-resistant Staphylococcus aureus infection in diabetic mice. J Med Microbiol. 2007;56(Pt 6):803-8.

13. Nidadavolu P, Amor W, Tran PL, Dertien J, Colmer-Hamood JA, Hamood AN. Garlic ointment inhibits biofilm formation by bacterial pathogens from burn wounds. J Med Microbiol. 2012;61(Pt 5):662-71.

14. Cutler RR, Wilson P. Antibacterial activity of a new, stable, aqueous extract of allicin against methicillin-resistant Staphylococcus aureus. Br J Biomed Sci. 2004;61(2):71-4.

15. Arzanlou M, Bohlooli S. Inhibition of streptolysin O by allicin - an active component of garlic. JMed Microbiol. 2010;59(Pt 9):1044-9.

16. An M, Shen H, Cao Y, Zhang J, Cai Y, Wang R, et al. Allicin enhances the oxidative damage effect of amphotericin B against Candida albicans. Int J Antimicrob Agents. 2009;33(3):258-63.

17. Yousuf S, Ahmad A, Khan A, Manzoor N, Khan LA. Effect of garlicderived allyl sulphides on morphogenesis and hydrolytic en- 
zyme secretion in Candida albicans. Med Mycol. 2011;49(4):444-8.

18. Klein E, Smith DL, Laxminarayan R. Hospitalizations and deaths caused by methicillin-resistant Staphylococcus aureus, United States, 1999-2005. Emerg Infect Dis. 2007;13(12):1840-6.

19. Sista RR, Oda G, Barr J. Methicillin-resistant Staphylococcus aureus infections in ICU patients. Anesthesiol Clin North America. 2004;22(3):405-35.

20. Jefferies JM, Cooper T, Yam T, Clarke SC. Pseudomonas aeruginosa outbreaks in the neonatal intensive care unit--a systematic review of risk factors and environmental sources.J Med Microbiol. 2012;61(Pt 8):1052-61.

21. Sjoberg BM, Torrents E. Shift in ribonucleotide reductase gene expression in Pseudomonas aeruginosa during infection. Infect Immun. 2011;79(7):2663-9.

22. Amini S, Hottes AK, Smith LE, Tavazoie S. Fitness landscape of antibiotic tolerance in Pseudomonas aeruginosa biofilms. PLoS Pathog. 2011;7(10):e1002298.

23. Perumal D, Lim CS, Sakharkar KR, Sakharkar MK. Differential genome analyses of metabolic enzymes in Pseudomonas aeruginosa for drug target identification. In Silico Biol. 2007;7(4-5):453-65.

24. Bjarnsholt T, Jensen PO, Rasmussen TB, Christophersen L, Calum $\mathrm{H}$, Hentzer M, et al. Garlic blocks quorum sensing and promotes rapid clearing of pulmonary Pseudomonas aeruginosa infections. Microbiology. 2005;151(Pt 12):3873-80. 